Proceedings of the 2011 Winter Simulation Conference

S. Jain, R.R. Creasey, J. Himmelspach, K.P. White, and M. Fu, eds.

\title{
CPI MODELING: COLLABORATIVE, PARTICIPATIVE, INTERACTIVE MODELING
}

\author{
Joseph Barjis \\ Delft University of Technology \\ Jaffalaan 5, 2628 BX Delft \\ The Netherlands
}

\begin{abstract}
The complex business processes of modern enterprises require extensive modeling efforts, especially for the purpose of analysis and redesign. Often, the scope of modeling goes beyond the boundary of one process and captures all the processes in interrelation with each other and the business environment. In the enterprise context, the modeling activity comprises both intra-organizational and inter-organizational processes. Such extensive modeling is increasingly becoming a challenge without innovative approaches. In this paper, an innovative approach, called Collaborative, Participative, and Interactive Modeling (CPI Modeling), is discussed. As for the applicability of the approach, empirical evidence from a few case studies is also discussed in this paper. The main emphasis of CPI Modeling is on joint modeling sessions with an active collaboration and participation of the users (business process owners).
\end{abstract}

\section{INTRODUCTION}

Modeling and simulation are a central activity of analysts in virtually any project involving analysis and design of processes or systems. The roles of modeling are even more crucial in complex processes such as enterprise business processes. A model plays several important roles: as a visual mapping of reality in the form of a diagram, it fosters communication; as a compact abstraction, it allows coping with complexity; as being based on shared concepts, it facilitates shared understanding; as a conceptual model, it serves as a blueprint for simulation design; and it has many other advantages. Modeling and simulation enable analysts to test different design options and conduct virtual experiments for whether design specifications are met by using virtual prototypes rather than physical experiments (Sinha et al. 2001). In spite of these discernible advantages, enterprise modeling is far from being an integral part of analysis and design efforts. In this paper, we focus on modeling within the enterprise domain. Enterprise business processes present challenges that hinder modeling for certain reasons including the fact that processes are spread over different organizational units with the involvement of human actors. Furthermore, the subtle interaction with the environment, which is a characteristic of an enterprise delivering service to its environment, contributes to the complexity of its business processes. As a result, enterprise modeling becomes time consuming, modelers often deliver incomplete models causing different perceptions of the users and business process owners, or models rather mainly represent the modeler (analysts) point of view, and so on.

These challenges necessitate new approaches in the practice of modeling and simulation, where collaboration, participation, and interaction of a larger group of stakeholders are accomplished to derive more valid models in a more effective and efficient manner. What is proposed in this paper is aimed to trigger a discussion towards collaborative and participative modeling.

The proposed approach of Collaborative, Participative, and Interactive Modeling was initially discussed in (Barjis 2009). For reading convenience, we refer to it as CPI Modeling. The primary assumption of CPI Modeling is that process owners (end users) must be actively involved in the modeling ses- 


\section{Barjis}

sions. It is natural that the local participants (end users) can provide the most accurate knowledge of their enterprise business processes (Jørgensen 2004).

The initial publication of the concept of CPI Modeling (Barjis 2009) put forward a few hypotheses by stating that this approach has several advantages such as expedited and accurate modeling, shared understanding, and an increased sense of model ownership and confidence in it by the end users. Further research, reported in this paper, focused on the proof of concept through case studies and feedback. In total, three case studies on three different enterprises have been conducted. All the three case studies followed the same format. They were conducted in a half-day session with participation of senior employees of the concerned enterprises. For each session, a basic sketch of the business processes was prepared prior to the session to use it as a starting point for modeling. That means that a prior visit to the company was made by a group of 3-4 graduate students to prepare a basic sketch and description of business processes. Based on such an initial input, the CPI Modeling sessions were facilitated by trained facilitators and an intuitive modeling language was used for creating models.

Before proceedings, it should be noted that the term of end user, participant, senior employees or managers are used synonymously. They all refer to what is also called business process owners - people with immediate knowledge of business operation in an enterprise.

\section{THEORETICAL DISCUSSION}

Enterprise engineering is an umbrella term for many research activities within the enterprise domains that refer to design, redesign and reimplementation of an enterprise or its business processes. Enterprise modeling implies alternative designs of envisioned business processes and comparison and critical analysis of suitable or acceptable design prior to implementation. At the same time, each design alternative is a product of consensus and shared understanding of different stakeholders. For both shared understanding and analysis of alternative options, modeling and simulation have been established as an integral to success and have been increasingly adapted in enterprise engineering. On the other hand, modeling and simulation are integral to each other as they complement towards the same outcome - communication, consensus, commitment, solution space exploration, etc.

When conducting enterprise modeling or proposing changes, different stakeholders may have their own priority or point of view. Without a laboratory to test each of the solutions or point of view, it might become a challenge to reach a consensus. Simulation is one of the tools allowing to test multiple options and highlight the advantages and drawbacks of each option. Through simulation not only a shared understanding can be better accomplished but also a consensus can be reached as the consequences of each option will be obvious beforehand. Especially, in multi-actors environments where politics or weight of stakeholders might play a superior role, simulation contributes to a more fair treatment of each option and consensus building. Often it may happen that a combination of two or more solutions results in a better outcome - a best of breed type of approach.

Below, we discuss modeling and simulation practice in an evolutionary manner - moving from a single person deriving enterprise models to collaborative, participative and interactive simulation activities.

Due to a number of issues in the conventional approaches, including modeling and simulation costs, demands for faster simulation, comprehensive simulation, researchers have been working on numerous innovative ways and approaches to elevate modeling and simulation practice. One such an approach is collaborative simulation, requiring an extensive interaction of different roles in the overall simulation project. Research on collaborative modeling and simulation has attracted researchers from different angles and perspectives (Sierhuis and Selvin 1996; Maghnouji et al. 2001; Taylor, Robinson and Ladbrook 2003). Overall, collaborative modeling and simulation is well accepted for its effectiveness and efficiency.

However, collaboration of analysts and simulation experts are not the only remedy needed for the vast challenges that enterprise engineering projects pose. The practice of simulation has also been challenged by a host of factors from the users' perspective - the problem owners' perspective. These factors, for example, include trust and acceptance of simulation models, transparency of simulation models and espe- 


\section{Barjis}

cially transparency of the decision making variables implemented in the model, etc. These factors prompted to the introduction and application of participative simulation. (van der Zee 2007) Participative simulation not only results in a more accurate and complete simulation model due to the participants that provide domain knowledge, but it also allows to validate models and, even more, enable the users to better understand their own domain and reflect on the reality they interact in. (Bok 2007) The trend of participation is further strengthened in treating the client, for whom the solutions are designed, as clientdesigner. In this role, the client is participating in the design process as a designer, although it is not a smooth and easy way for the client partners to enter the design world. (Weedman 2008)

The current trend in the practice of simulation, however, moves to a completely different level and experience, although with rather more challenges than successes. This trend is characterized as interactive simulation. Although the need for interactive simulation has been discussed for a long time (Bell and O'Keefe 1987), the current understanding of interactive simulation is from completely different perspectives. Interactive simulation, unlike the notions of collaborative and participative simulation, has a wide interpretation. It is interpreted in different ways from visualization of simulation model and outcomes (Macal 2001) to a true interaction with the model for decision making purposes or visually scenarios testing, to, actually, groupware tools. (Filho, Hirata and Yano 2004) In all interpretations, interactive simulation is the most promising and most challenging trend and comprises a potential future for simulation research.

In the following section, we put the notions of collaboration, participation and interaction into a whole with interrelation as a holistic approach. We propose and discuss the approach of Collaborative, Participative, and Interactive Modeling, or CPI Modeling for short.

\section{CPI MODELING FRAMEWORK}

In the enterprise context, the modeling activity requires to model business processes in regard to intraorganizational as well as inter-organizational processes. Such a broad modeling scope requires innovative approaches to ensure effective and efficient modeling. As mentioned earlier, one such an approach discussed in this paper is Collaborative, Participative, and Interactive Modeling (CPI Modeling), where modeling is conducted in a joint session(-s) with collaboration and participation of the stakeholders.

The objective of CPI Modeling is to collaboratively create a trustworthy and complete essential model of an enterprise with the participation of the end users using an intuitive modeling language, where the model is validated by the end users (process owners). The modeling process is facilitated by a professional facilitator, because facilitated modeling interventions better ensure communication, consensus and commitment (Rouwette 2011).

The comprehension of an enterprise model is very important for acceptability, usability, and more importantly, trust in the model and its results. CPI Modeling should result in quick modeling and trustworthy models for many reasons among which the following two are the most prevailing:

- Having onboard all competent end users eliminates the need for iterations to get complete information and resolving conflicting views.

- Trust in the derived models, as a result of participation in the process, reduces validation and verification efforts. Furthermore, the participation of end users fosters implementation of conclusions (Rouwette et al. 2011).

CPI Modeling is a top-down approach in model abstraction starting from a high level of abstraction and moving towards a more detailed description of enterprise business processes. 


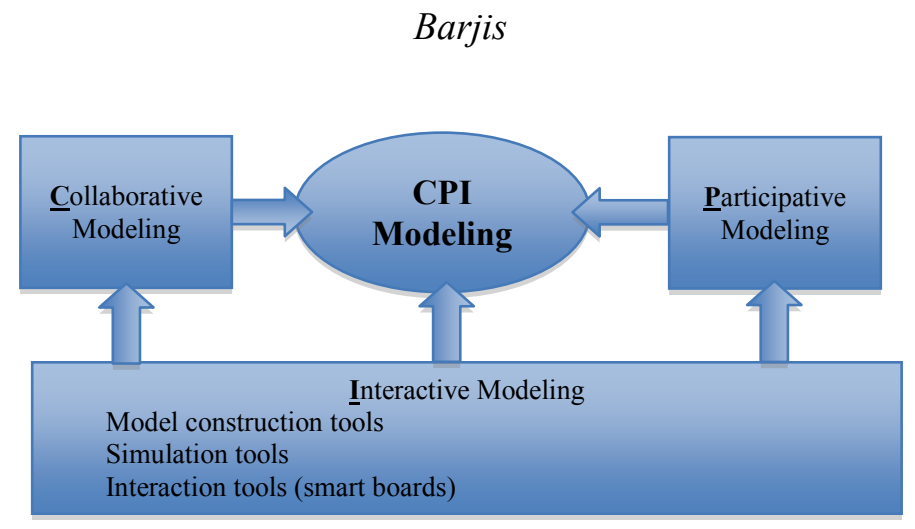

Figure 1: CPI Modeling Framework

The notions of 'Collaboration', 'Participation', and 'Interaction' are the three constituents of success for complete, accurate, and fast analysis and design in complex enterprise settings. The CPI Modeling approach consolidates three aspects of complex processes modeling as illustrated in the diagram of Figure 1 -the expert aspect, the user aspect, and the tool aspect:

- Collaboration - Expert aspect - emphasizes cross-disciplinary collaboration of business analysts, modeling experts, facilitator to organize modeling sessions.

- Participation - User aspect - emphasizes participation of the business process owners, managers, stakeholders that provide input for modeling. One reason why users should be involved in creating models is that users in modern enterprises possess increasingly more knowledge and maturity of their processes and capability of IT, which contribute to effective modeling. Furthermore, now users have more power of acceptance or refusing organizational changes or innovations so their involvement creates commitment.

- Interaction - Tool aspect - emphasizes tools for creating models and technologies for enabling collaboration.

Below we discuss each aspect of the CPI Modeling in more details.

\subsection{Collaboration}

Collaboration has been seen as a way that results in fast and accurate model development when modeling complex business processes of modern enterprises. But collaboration itself is a subject of engineering approach, where explicit guidelines have to be designed for facilitating collaboration step by step.

Collaboration also requires guidance and orchestration by an experienced facilitator. Facilitators with modeling experience and basic domain knowledge are more successful in leading the participants. A facilitator plays an important role in the CPI modeling sessions. He sets a convenient atmosphere resulting in a collaborative, participative and interactive process. He should be in control and prevent chaos in group discussions and keep up the speed and progression. This will eventually lead to a more accurate, validated and verified model. He should be competent of providing explicit opinions because that might influence the process. He should be supported by a crew to operate devices such as a smartboard and other presentation solutions.

Finally, collaboration requires that modelers (with knowledge of modeling language and techniques) and analysts (with expertise in analysis and modeling) are collaborating to design accurate models. The objective of collaboration is creating a complete enterprise model.

\subsection{Participation}

Involving the employees of an enterprise in creating a model of their daily work is very important. Often senior or managerial level employees are the most suitable, as they know business processes across the 


\section{Barjis}

whole unit. While more participants will provide more insight, practice also shows that this might slow down the modeling process. So the number of participants should be proportionate to the size and scope of the modeled enterprise.

Often, enterprise modeling and simulation activities are undertaken for possible improvements and changes in the current practice, organizational structure, or alignment with new technology. It is hard to achieve these goals without participation and approval of stakeholders.

Often, also, the goal of modeling and simulation is to document the operations and business processes, and create shared understanding of what and how an enterprise is operating. This shared understanding, which is achieved through a complete picture of an enterprise, would be difficult without the participation of key employees who have first hand knowledge of their daily works.

Often, models convey different accounts of the studied situation (business processes or systems) from different perspectives or stakeholders accounts. Numerous iterations of modeling and simulation are required to build accurate models, which makes the process tedious, extensive and costly. Consolidation of different accounts and expedition of the modeling process are another challenge that is hard to cope with unless the modeling involves participation of the key users.

Finally, the participation of the enterprise contributes to the validation and verification of the models, especially when simulation methods are deployed in the process.

The objective of participation is provision of input information for creating models, validation of models, and instilling sense of ownership of the models.

\subsection{Interaction}

The interaction part of CPI modeling is rather the most challenging as it can cover multiple aspects. First of all, innovative tools (technologies) are needed to provide a medium for creating and drawing models. Large interactive smart boards are examples of such tools. There are special software developed for smart boards that supports editing, modifying, storing, and transmitting models. A more advanced tool should allow multiple modelers and users to participate in the model creation at the same time. This fosters a sense of ownership and further motivates active participation. Also advanced tools should furnish interaction of modelers, analysts, and participants during the modeling process.

Tools used for interaction should be intuitive and powerful to create complex models.

The next level of using a tool is to create dynamic models. While static models can serve many purposes, dynamic models may be created for more advanced analysis and design efforts. For conducting tests, comparing various design options, introducing improvements, and discussing scenarios, the tool must support simulation of models and allow easy intervention and manipulation of the models.

The objective of interaction is to support collaboration, facilitate simulation, and automate the modeling process.

\subsection{Roles in CPI Modeling}

Time is hard to manage during the sessions due to the multiplicity of participants of CPI Modeling. By designing procedures and guidelines, the time needed might be reduced. Dividing roles might also save time in the process.

The participants have been divided in three categories: Users (company representatives), Analysts (facilitation crew) and Observers.

\section{End Users}

- Senior employees with direct knowledge of the business processes

- Managers of business units with expertise about the process goals and the influence of the process (and changes) on the company as a whole. Multiple managers may be needed to cover all processes. They should be involved to make decisions on model design and changes. 


\section{Barjis}

- Process manager, if applicable, with expertise and know-how about the processes that will be modeled.

- IT/IS specialists. If the company has its own IT department, a high player of the IT staff should join the session to bring IT perspective to the CPI Modeling session and also experience the techniques used in the CPI Modeling.

\section{Analysts}

- The Coordinator handles communication with all the participants to keep the communication clear for the company and be the only one who will ask direct questions about the company, use consistent language (terminology), estimate approximate time needed for CPI session, follow up communication, etc. This role could be combined with the role of facilitator or secretary.

- The Facilitator can be considered a communication expert who leads the discussion during the session to create models. He should make sure that rules of the CPI sessions are followed, adequate participation is accomplished, ambiguities are removed, a clear and complete model(-s) is created. The facilitator needs to be a person who has good social skills, so he can get people listen to him and to others. Next to this, the facilitator needs to have a good feeling for when to interfere with the process of modeling. One of his tasks is to introduce and explain the CPI technique to all participants.

- The Domain experts are people who have sufficient expertise about the subject to discuss (out of the box) ideas about business processes. This could be consultants, modelers, employees of the company, or IT people. They can give additional input and ask critical questions.

- The Modeler draw the models based on the description and discussion among the participants. He also adapts the model during the CPI session. It is suggested that the modeler creates an initial model based on preliminary visits, readings, and description of the company's business processes. The modeler should not only be able to work with the software to make clear overview of the proposed model, but also have knowledge about the hardware to keep the equipment working.

- The Secretary takes notes of what everyone is telling during the modeling session. Primarily, this results in a detailed description of business processes. This also includes data which doesn't seem important at the moment but can become useful during further interventions. Recording the session might also be very helpful.

\section{Observers}

A group of observers (domain experts, IT people, graduate students, researchers, consultants, tool developers, social or psychological science researchers), whenever available, can be asked to sit in the CPI sessions to observe the sessions and document the sessions from behavioral perspective. Their feedback should feed further research and improvement of CPI Modeling.

\section{CPI MODELING APPLICATION}

The proposed approach of CPI Modeling was tested in several enterprise modeling sessions carried out on different enterprises. The first case study was conducted in October 2008 and the last two in October 2010. In each CPI modeling session, all the required parties were invited and actively participated in creating enterprise models. These included managers of units from the concerned enterprises, enterprise modeling experts, and session facilitator. For reflection and observation, a number of graduate students were also invited. The empirical data from using CPI modeling yielded interesting results, which we will discuss later.

In the conducted CPI Modeling sessions, the focus of research was on collaboration and participation using interactive smart boards. As for the interaction aspect, it has not yet been studied due to a lack of access to proper tools and also training for conducting interactive sessions.

One case study was conducted in DutchPlast BV, which we describe in details to understand the format of CPI Modeling. 


\section{Barjis}

\subsection{Case Study: DutchPlast BV Enterprise}

This case study was conducted on DutchPlast BV (disguised name), a plastic production company, located in Westland-Area, The Netherlands. The company was launching an initiative to review its business processes and improve the current processes with reducing delays, and developing a new information system that will support the redesigned business processes.

We organized a half-day session with the following participants:

- $\quad$ End users - from DutchPlast, the technical director and order and procurement director - business process owners with high expertise and knowledge of the enterprise operations.

- Enterprise modeling expert - an author of enterprise modeling methodology

- Modelers - expert modelers.

- Facilitator - a professional collaboration facilitator

- Observers - a group of observers to document the session, including a group of graduate students who previously have taken an enterprise modeling course.

The modeling session was conducted on a large interactive smart-board allowing use of electronic color pens on a touch-screen. The set-up used for the modeling task is visualized in Figure 2.

The modeling covered the whole enterprise business processes from placing order for a new product to manufacturing, delivering and transporting the product, and payment handling. The model also covered the processes with inventory control and purchase of supplies and materials.

In the beginning of the session, we explained the modeling approach to the participants and created a small model to illustrate the approach and explain the key concepts and constructs used for creating a model. We only modeled the business transactions, actor roles and results of each business transaction to keep the model at the highest abstraction level.

We kept the modeling notations to 2-3 elements (box, arrow, swim-lane), but enhanced them with color to visually distinguish between activities and the results they create. To support the collaborative modeling effort, we used an electronic whiteboard with Smart Ideas ${ }^{\mathrm{TM}}$ software. This tool allowed us to capture the model during the collaboration process in a way that is readable for all participants and flexible with respect to corrections and layout. Given the size of the model, a normal whiteboard or flip-over would have been full quite quickly and difficult to read. Furthermore, when making changes or corrections, the model would become messy. Using the interactive whiteboard, the model could be modified and stored digitally.

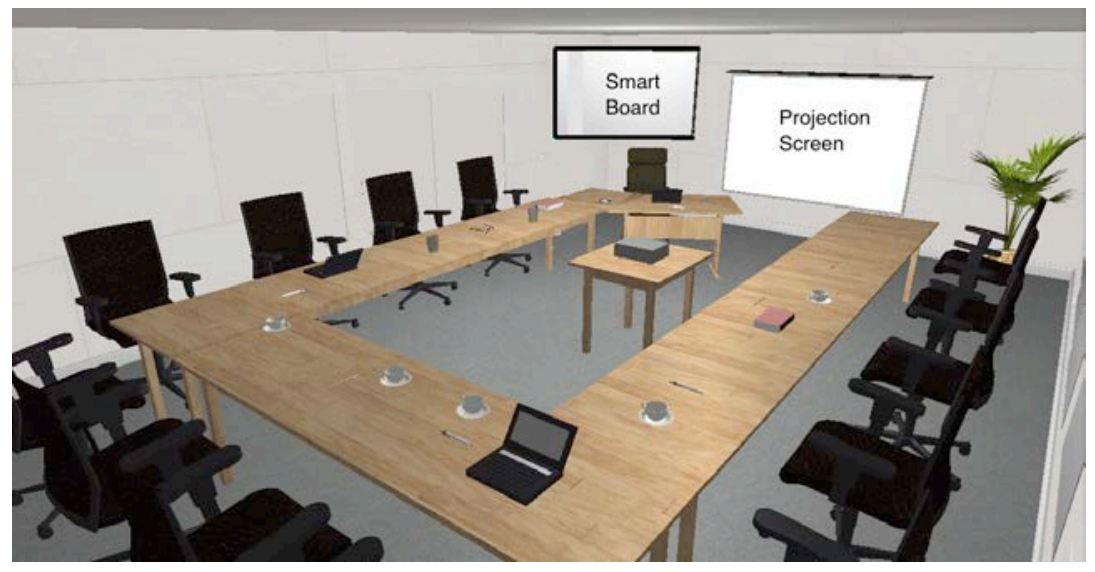

Figure 2: CPI Modeling set-up

In a similar fashion as the DutchPlastic cases study, two more case studies were conducted. The result of each case study was a list of business transactions, associated actor roles, and results that each business 


\section{Barjis}

transaction creates. All business transactions and actor roles were depicted in a diagrammatic model. The resulting model was reviewed for validation purpose following the face validation approach.

\section{DISCUSSION}

In all CPI Modeling sessions, observers (graduate students with knowledge of enterprise modeling) were asked to write feedback and reflection on the modeling sessions. In total, some 60 feedbacks were collected based on three cases studies. The feedbacks were based on open questions. They reflected on the CPI Modeling approach in general by highlighting whatever they deemed important from modeling perspective. This section contains a summary of the feedback and reflections consolidated around a few related aspects of CPI Modeling.

The most important finding was that it has been demonstrated that CPI Modeling results in a faster and more accurate enterprise modeling. As stated, three CPI Modeling sessions have been conducted and each session took a half-day.

The following are other major points of the feedbacks and reflections, mainly in the language of graduate students who participated as observers.

In traditional approaches to enterprise modeling, the modeler seems to take the leading role by, e.g., preparing questions for an interview or survey, observing employees, or deriving models from existing documents. They also seem to have a more dominating role in the process, because they decide what to include in the final model. With CPI modeling process owners (the company representatives) take part in this process to think along with the modeler about the processes, because they have detailed knowledge about the processes in the company and the company organization. A CPI modeling session fosters direct feedback. This will result in a discussion and will provide additional information to the modelers. This close and active interaction of the modelers and the company representatives is an advantage of CPI modeling compared to the traditional approaches. It improves the quality of the input and fewer assumptions are made.

Possible faults or misperception can be discovered immediately, which saves time and effort as well as prevents possible propagation of the errors. This way less meetings and individual sessions are needed compared to traditional approaches and a more efficient process can be achieved. This will reduce modeling costs and duration.

CPI modeling improves the understanding of the company representatives. This will have two effects; more enthusiasm of the company and more support to get the design implemented. If the company is more enthusiastic, it is also more likely to share sensitive information, because they will become aware that the completeness of information is a critical success factor of the model. To get the design implemented support of the company is needed and therefore it is important that the company is committed to the process and results.

The modelers and company representatives are not the only participants in a CPI session, but there are also consultants, analysts and cross-disciplinary expertise. This stimulates the creativity because they are more likely to give new insights which the modeler or company representative wouldn't have thought of themselves. In fact, the problem is observed from multiple angles.

CPI modeling makes it easier to validate and verify models by the company representatives, because they are present during the sessions and can provide face validation.

The core strength of CPI modeling is the possibility to go through the model step by step and make changes where necessary. The disadvantage of it might be that too much time will be spent on points of no interest. Start with a simple model then move on to a more extended model. For doing so, a generic set of guidelines should be developed and tested.

All participants should be able to follow the steps. Towards this end, tools and technology (shared smartboards) and adequate software are crucial. Working with tolls requires a trained person.

The tools used for modeling should be easy to use, follow, and understand for all the participants, even those with no experience in modeling; nevertheless the tools should also be able to capture complex enterprise processes and behavior. This will lead to more effective and efficient and thus successful use of 


\section{Barjis}

the CPI Modeling. For advanced analysis, simulation is a crucial complement. Therefore, the modeling method and language used need to be balanced between ease of use and understanding on one hand usability for advanced analysis on the other hand.

\section{CONCLUSION}

In this paper, we attempted to introduce an innovative approach for modeling complex business processes in an enterprise, where emphasis is put on the participation of the end users (business process owners) during the modeling.

CPI Modeling can be best achieved if the participants are in one location and interact synchronously. By this, several objectives of CPI Modeling can be achieved, including expedited model design, validation, consensus building, etc.

However, the collaborative, participative, and interactive modeling approach, as introduced in this paper, is in its early infancy - conception phase. This approach requires further research and more case studies to gather empirical insight. At this stage, this approach only opens up an interesting and challenging research direction in the field of enterprise modeling and simulation.

However, it should be noted that in the case studies conducted so far, the simulation part was not included. The challenge with the simulation is lack of adequate tools that will allow a speedy simulation model design based on the simplistic model created during the CPI session. This is an interesting challenge for future research.

\section{REFERENCES}

Barjis, J. 2009. Collaborative, Participative and Interactive Enterprise Modeling. In Filipe, J., Cordeiro, J. (Eds.) Enterprise Information Systems. Lecture Notes in Business Information Processing, Volume 24, Springer-Verlag, Berlin Heidelberg.

Bell, P.C. and R. O'Keefe. 1987. "Visual Interactive Simulation - History, Recent Developments, and Major Issues." Simulation, 49 (3): 109-116.

Bok, B.M. 2007. "Experiential Foresight: Participative Simulation Enables Social Reflexivity in a Complex World." Journal of Futures Studies, November, 12(2): 111 - 120

Filho, W. A., C. Hirata and E. Yano. 2004. "GroupSim: A collaborative environment for discrete event simulation software development for the World Wide Web." Simulation, 80 (6): 257-272.

Jørgensen, H.D. (2004). Interactive Process Models. PhD dissertation, Department of Computer and Information Science, Norwegian University of Science and Technology Trondheim, Norway, January 7, 2004

Macal, C. M. 2001. "Simulation and Visualization.” Simulation, 77(3-4): 90 - 92.

Maghnouji, R., G. de Vreede, A. Verbraeck and H. Sol. 2001. Collaborative Simulation Modeling: Experiences and Lessons Learned. In: HICSS 2001: Proceedings of the 34th Annual Hawaii International Conference on System Sciences (HICSS-34), vol. 1, p. 1013. IEEE Computer Society, Washington

Rouwette, E.A.J.A. 2011. "Facilitated modeling in strategy development: measuring the impact on communication, consensus and commitment." The Journal of the Operational Research Society, 62: 879887.

Rouwette, E., H. Korzilius, J. Vennix, and H. Jacobs. 2011. "Modelling as persuasion: The impact of group model building on attitudes and behavior." System Dynamics Review, 27(1): 1-21.

Sierhuis, M. and A. Selvin. 1996. "Towards a Framework for Collaborative Modeling and Simulation." In Proceedings of the 6th Computer Supported Cooperative Work Conference.

Sinha, R., C. Paredis, V. Liang and P. Khosla. 2001. "Modeling and simulation methods for design of engineering systems." Journal of Computing and Information Science in Engineering, 1 (1): 84-91.

Taylor, S.J.E., S. Robinson and J. Ladbrook. 2003. "Towards Collaborative Simulation Modelling: Improving Human-to-Human Interaction Through Groupware." In Proceedings of the 17th European Simulation Multiconference (ESM 2003). SCS Europe Bvba, Erlangen, Germany, 474-482. 


\title{
Barjis
}

van der Zee, D-J. 2007. Developing participative simulation models-framing decomposition principles for joint understanding. Journal of Simulation (2007) 1, 187-202.

Weedman, J. 2008. "Client as designer in collaborative design science research projects: what does social science design theory tell us?” European Journal of Information Systems, 17 (5): 476-488.

\begin{abstract}
AUTHOR BIOGRAPHIES
JOSEPH BARJIS is an Associate Professor of Systems and Modeling \& Simulation at Delft University of Technology. Dr. Barjis research interests are focused on business process modeling and simulation, enterprise modeling and simulation, healthcare processes modeling and simulation, information systems design, system analysis and design, collaborative, participative, and interactive modeling (CPI Modeling). $\mathrm{He}$ is the founder of the Special Interest Group on Modeling and Simulation (SIGMAS) of the Association for Information Systems (http://www.AIS-SIGMAS.org/); he is also the founder of the International Workshop on Enterprise \& Organizational Modeling and Simulation (http://www.EOMAS.org/); he is a member of Program Committee and Editorial Board in several international conferences and journals. More information at: http://www.JosephBarjis.com
\end{abstract}

